\title{
SOCIAL NETWORKS OF SPORT AND THEIR POTENTIAL IN SMART URBAN PLANNING PROCESSES
}

\author{
RAQUEL PÉREZ-DELHOYO' ${ }^{1}$, HIGINIO MORA², RUBÉN ABAD-ORTIZ1 \& RAFAEL MOLLÁ-SIRVENT² \\ ${ }^{1}$ Department of Building Sciences and Urbanism, University of Alicante, Spain \\ ${ }^{2}$ Department of Computer Technology and Computation, University of Alicante, Spain
}

\begin{abstract}
Information and data have become a new working tool for many disciplines including urbanism. Its incorporation into the field of urban planning is currently a process with great development potential. Within this context, citizens are one of the most important sources of data, providing relevant information for better smart city planning, adapted to their preferences and needs. In this sense, social networks are very powerful tools that city planners have to know directly from users the use they make of public space. It is clear that this information cannot be left out of the process of smart planning and design of today's cities. Specifically, this work focuses on the study of sport social networks and aims to determine which sport social networks offer the greatest potential for improving urban planning processes. To this end, the main existing social networks in this field are studied and, as a conclusion, the advantages and disadvantages that make these sports networks an opportunity to move towards smarter, more participatory and inclusive urban planning are discussed.

Keywords: citizen participation, citizen-centric urban planning, inclusive city, smart city, smart urban planning, social networks of sport, technology-aided urban planning.
\end{abstract}

\section{INTRODUCTION}

Urban planning is currently clearly defined by the need for a reformulation of the way in which the practice of this discipline are developed, in order to improve the way in which cities evolve to respond to the needs and preferences of their inhabitants [1]. In this reformulation, the Information and Communication Technologies (ICT) are the main lines and guidelines for change. The emergence of this type of technologies for sharing information, with a clear expansion in the last 10 years, has led to a new way of understanding and living the city. The significant boom in the use of portable smart devices such as mobile phones by citizens and the large number of Internet-based tools available have been the main causes of this change. Greater connection and social participation in the life of cities is made possible by these technologies [2]. This new way of conceiving and planning the city, more participative and inclusive, which applies ICT, responds to the idea of smart urban planning which, in turn, is included within the concept of smart city. A smart city allows citizens to interact with it in a multidisciplinary way and adapts in real time to their needs efficiently, through the innovative integration of infrastructures with smart management systems [3].

A good example of the application of these technologies to promote the participation of citizens is social networking. In the field of urban planning, the analysis of data retrieved from social networks makes possible new ways of addressing urban issues and intervening in urban public space in a much more flexible and immediate way compared to the greater rigidity of other traditional tools of urban planning. The ease of adding geographic information to these social networks has led to a substantial increase in the amount of information of this type available free of charge. That is why one of the most interesting challenges for current urban planning is to be able to analyse this information, which is generated by citizens on a voluntary basis, in order to find answers to questions such as the places visited or the patterns of behaviour and thus adapt to their preferences and needs. 
Specifically, this work focuses on the study of sport social networks and aims to determine which ones offer the greatest potential for improving urban planning processes. To this end, the study of the main social networks existing in the field of sport and the discussion of the advantages and disadvantages that make these sports networks an opportunity for urban planning is presented in this work. The main objective of this work is, therefore, to get to know the main characteristics that make some sports social networks valuable sources of information to aid decision-making processes on issues related to urban planning. This knowledge will enable city planners to move towards more smart, participatory and inclusive urban planning.

The rest of the work is structured as follows: in Section 2, the methodology is briefly described; in Section 3, a series of eight sport social networks is studied, and some previous related work is collected; in Section 4, a comparative study of these social networks is carried out and the advantages and disadvantages that make them an opportunity for urban planning are discussed; and finally, in Section 5, some conclusions are drawn.

\section{METHODOLOGY}

The methodology used to achieve the objectives consisted of three phases: (1) A first phase of selection of the most relevant social networks existing in the field of sport. This selection has been made on the basis of popularity criteria. (2) A second phase of study of the selected networks. For the particular study of each of the networks, both the web services they offer and the applications for mobile devices associated with these web services have been analysed. (3) A third phase of comparative study in order to discuss the advantages and disadvantages that make these networks an opportunity for urban planning. The study has focused mainly on networks that offer geographic information and geolocated spatial data.

\section{STUDY OF THE MAIN SOCIAL NETWORKS OF SPORT}

Sporting social networks relate the data of the routes made by the athletes with the factors that allow them to compete with each other, such as the time spent or the difficulty of the routes. This is the key principle on which the different types of sports social networks and their associated applications are based. In this section, a series of these social networks of sport, both their websites and applications, are analysed in order to be able to identify which of them offer the greatest opportunities to move towards smarter, more participatory and inclusive urban planning. More specifically, this work focuses on the sport of running.

\subsection{Social network "Atleto"}

Atleto [4] is a sport social network that focuses on the creation of a user profile based on the skills and habits of sports practice, in order to be able to show the user a selection of specific activities always in a radius close to their location. In addition, it is also possible to connect with other athletes who are using the application and share and assess each other the exercises carried out. The character of this social network is very basic (Fig. 1). In Europe it is not very well known. However, its use is very important in the United States which is its country of origin. In this location, examples of very different use can be found, from fitness classes to activities such as yoga. The low international penetration of this social network is also due to its recent creation in 2015. A newly created social network is more difficult to disseminate when well-established alternatives already exist. Regarding what is of interest in this research, Atleto does not have geolocated data generated by users that can be retrieved, so that, by not providing information of this type, the social network does not offer a priori interest for urban analysis within the context of this research. 

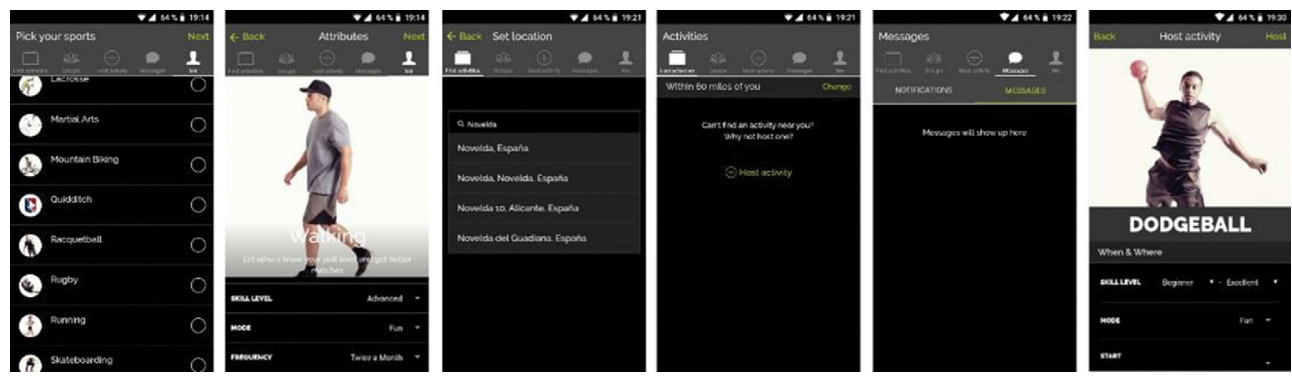

Figure 1: Social network “Atleto". View of some application screens (Source: Atleto).

\subsection{Social network "Gotzam"}

Gotzam [5] is a social network for athletes but also offers other services related to the organization of events. Its functionalities are related to organizational aspects of sporting events, such as event registration management, race timing, sports results management, as well as other services such as sports insurance, marketing or communication services.

This social network has no mobile application, so the web itself recommends the use of other applications, such as Runtastic, Runkeeper, Strava or Endomondo. However, it is possible to download the geolocated routes of the different events for analysis in GPX format. The potential of this social network, and the difference with other sport social networks studied, is the ability to inform users on the events that take place close to their location and that are related to the sporting activity they practice (Fig. 2).

\subsection{Social network "Runkeeper"}

Runkeeper [6] is a social network for sports monitoring that was created in 2008 and has belonged to ASICS since 2016. The principle of operation of this social network is an application that is based on the location of the sports activities of users in the cartography of Google Maps. However, the cartography is only used as a support to the training plans. In addition, a connection to other monitoring applications, such as Google Fit, is included, but without any social intention.

The data generated with this application are related to the physical characteristics of athletes (weight, sex, etc.). These characteristics are fundamental to improve their training and
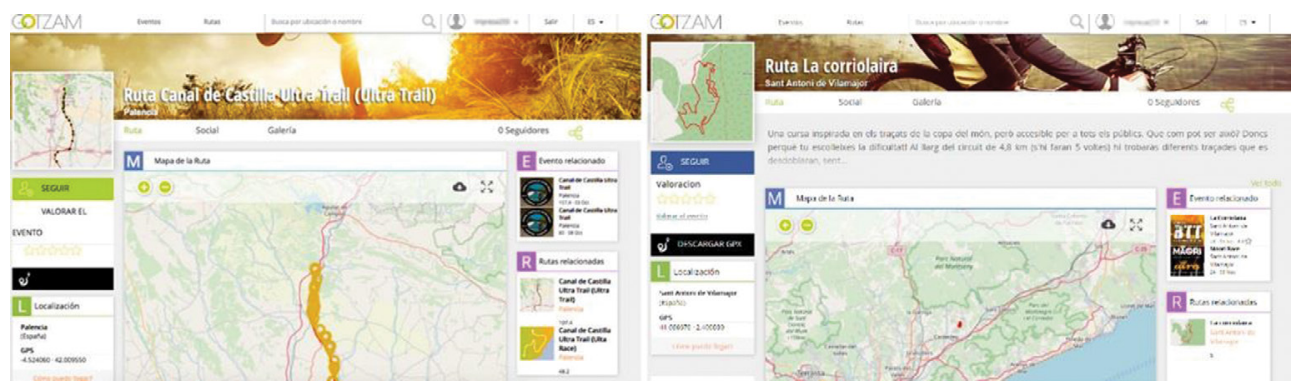

Figure 2: Social network “Gotzam”. Examples of routes on the web (Source: Gotzam). 

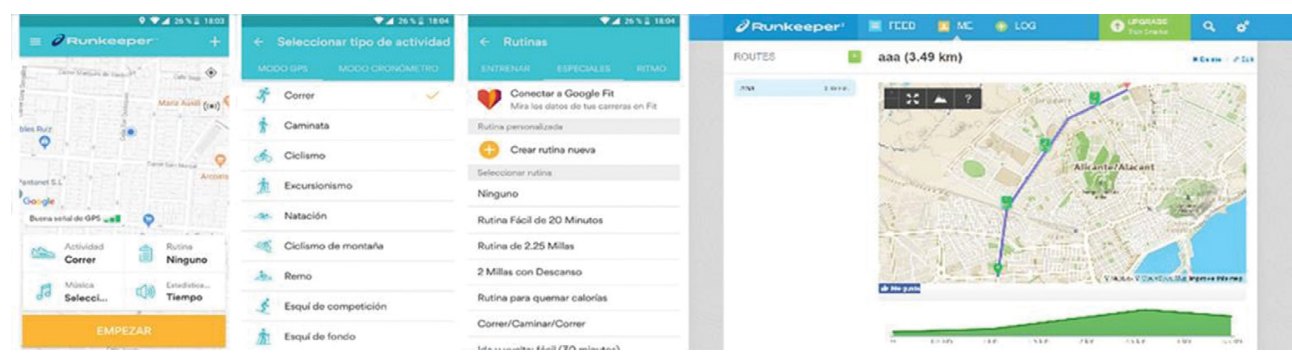

Figure 3: Social network "Runkeeper". View of some screens of the application and the interface to visualize routes on the web (Source: Runkeeper).

performance. In addition, users have the opportunity to make comments after their training and check whether they have achieved the proposed objectives.

Thus, the main attraction of this tool is the detailed analysis of statistics of the users' sporting activity. Although it is possible to compare the data obtained with that of other users, the potential of this social network is to create personalized training plans. In addition, the application has a gambling part, consisting of completing a series of challenges, which change over time in order to increase user motivation.

Regarding what is of interest in this research, it should be noted that it is not possible to obtain routes in geographical formats. It is only possible to visualize the routes and compare the times used (Fig. 3). Therefore, the social network does not offer a priori interest in the context of this research. With respect to the scientific literature, it has focused on analysing issues such as effects on health [7], and the impact of social sports networks in general [8,9]. The strong point of this application is to help the user to keep a precise control of his physical activity, so the studies carried out have to do with this issue.

\subsection{Social network "Sports Tracker"}

The social network Sport Tracker [10], created by Nokia in 2004, it was a pioneer in GPS tracking. It consists of a website and an associated application. The main functionality of this social network is to analyse the routes taken by athletes on a cartographic basis, offering data such as time, distance covered and other factors such as the speed reached and the difference in altitude of ascent or descent of the route.

Through the mobile application, the data are obtained and then analysed and presented on the web. Sports Tracker has a route search engine on map and it is possible to compare the records obtained with those of other users. Thus, it is possible to identify the points where sports performance can be improved (Fig. 4). In addition, once the sports activity has been carried out and a route has been registered, it is possible to share it in other social networks.
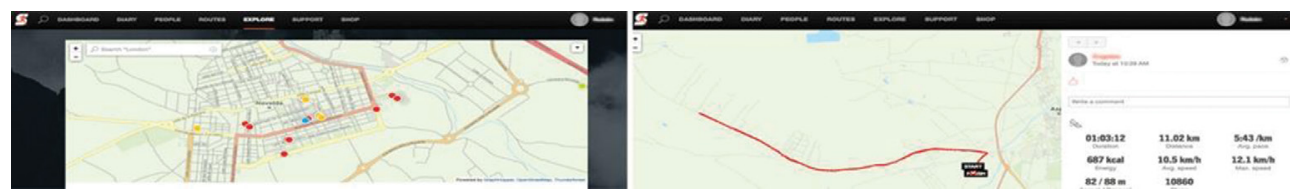

Figure 4: Social network "Sports Tracker". Search and route information on the web (Source: Sports Tracker). 
It should be noted that geolocated data can be retrieved from this social network in GPX format. The greatest attraction of this application is its important development in recent years, which has led to the emergence of some specialized research [11].

\subsection{Social network "Runtastic"}

Runtastic [12] was created in 2009. Initially, this social network was based on the collection of data from athletes dedicated to sport in a playful way. Nowadays, it offers many more possibilities. It consists of a series of applications that share design and functionality and that form an ecosystem for measuring sports training.

The main features of Runtastic are as follows:

- Geolocated record of the sporting activities performed through the mobile application: from the geolocation platform of Google Maps, the user's activity is tracked and the result is shown when it is finished.

- Connection with the most popular social networks: through networks such as Facebook or Twitter, it is possible to establish comparisons with the exercise developed by other users, thus promoting a competitive aspect that helps greater use of the social network. This same functionality is also available on the own website.

- Motivation-based training plans: motivational messages and photographs of events are shown. Users are also given the opportunity to sign up for these events.

The social network Runtastic is very focused on the collection of data. As an important quality, its complete interface for the creation and management of routes should be highlighted. It is possible to filter routes based on a multitude of factors, such as distance covered, time spent and places visited. This facilitates a complete analysis of the routes (Fig. 5).

Runtastic also allows routes to be shared easily, in order to be able to make comparisons with the activities carried out by other users. There is a simple interface for searching for routes, both by geographical location and by route name, showing as a result very complete information.

In addition, it is possible to download the route map in vector format for import into a geographic information system. However, it must be taken into account that there is no API
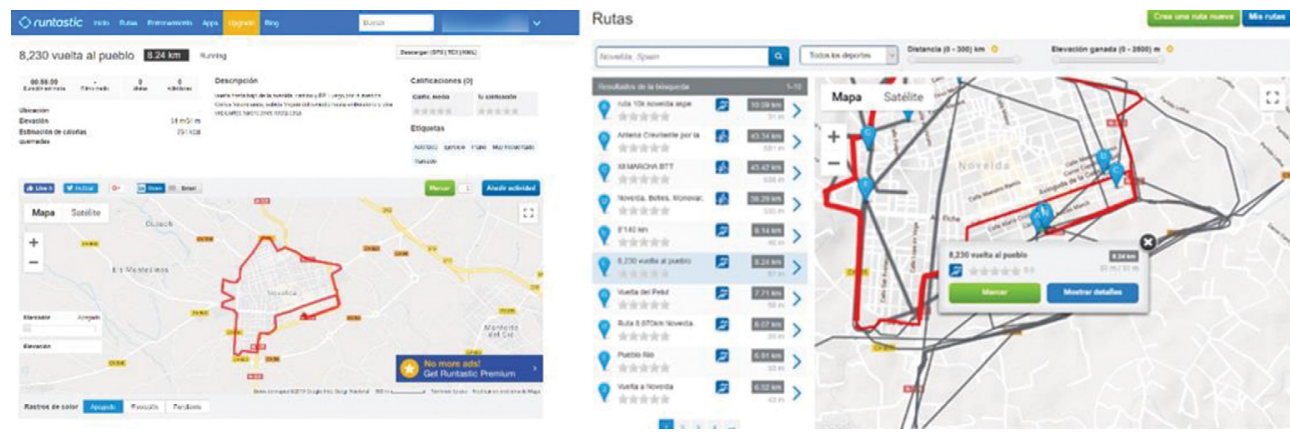

Figure 5: Social network Runtastic. View of different screens of the website: route visualization and search tool (Source: Runtastic). 
(Application Programming Interface) to work with the data through the platform, and only manual actions can be carried out on each route obtained through the maps.

In regard to Runtastic, some scientific publications have been carried out [13].

\subsection{Social network "Endomondo"}

Endomondo is a social network that is part of a group of applications and tools belonging to the company Under Armour. The most important applications are MyFitnessPal; Under Armour (UA Record and UA HealthBox); and MapMy applications (MapMyRun, MapMyFitness, MapMyRide, MapMyWalk and MapMyHike), which record users' sports activities. These applications are interrelated in their features but are not integrated, i.e. there is no data interaction between them.

Endomondo allows users to create personalized training plans, follow maps and share training with other users [14]. The social network is linked to an application for mobile platforms that collects, at all times, the user's sporting activity when exercising. It focuses mainly on cycling and running activities but also includes other sports. Its user interface basically shows the routes covered by users, the times used and their progression. As soon as users complete the routes, they are presented with a results summary screen, in which the main parameters of length, distance covered, health effects and others parameters are described.

Athletes are also offered with the opportunity to share their impressions of the exercise performed, as well as to share the routes covered, through the most popular social networks. In addition to this, there are other options that are not free, with which it is possible to have access to other factors that affect the training, such as weather information, performance graphs or connection to external devices.

Complementing this core functionality, this application has a social interaction component, which is based on feedback through a notice board and the creation of events. It is possible to add friends in order to follow their trainings and compare the results. In addition, through events, in which users of the application from different parts of the world participate, it is possible to compete through the visualization of routes and basic training parameters.

The web version (Fig. 6) includes, in addition to the features listed above, the creation of personalized routes. It also includes the creation of personalized training plans in a more exhaustive way, although this option is not free of charge.

It should be noted that the most important variable that highlights in this social network is in regard to the user community and its integration with the most popular general social networks [15]. It is possible to share data on Facebook and Twitter, for example, so that any user can see all the information about the content of this application without having to download it. In this sense, it is worth highlighting the studies that have been conducted in this regard, which have shown that it is possible to fully analyse the data obtained from this social network [16].

As for the interest of this research, Endomondo allows users the free download of geolocated routes generated by users, so the social network offers a priori an interest for urban analysis in the context of this research.

\subsection{Social network "MapMyRun"}

MapMyRun [17] is a social network of sports monitoring belonging to the company Under Armour since 2015. It is based on the registration of sports activities from the location. Since 

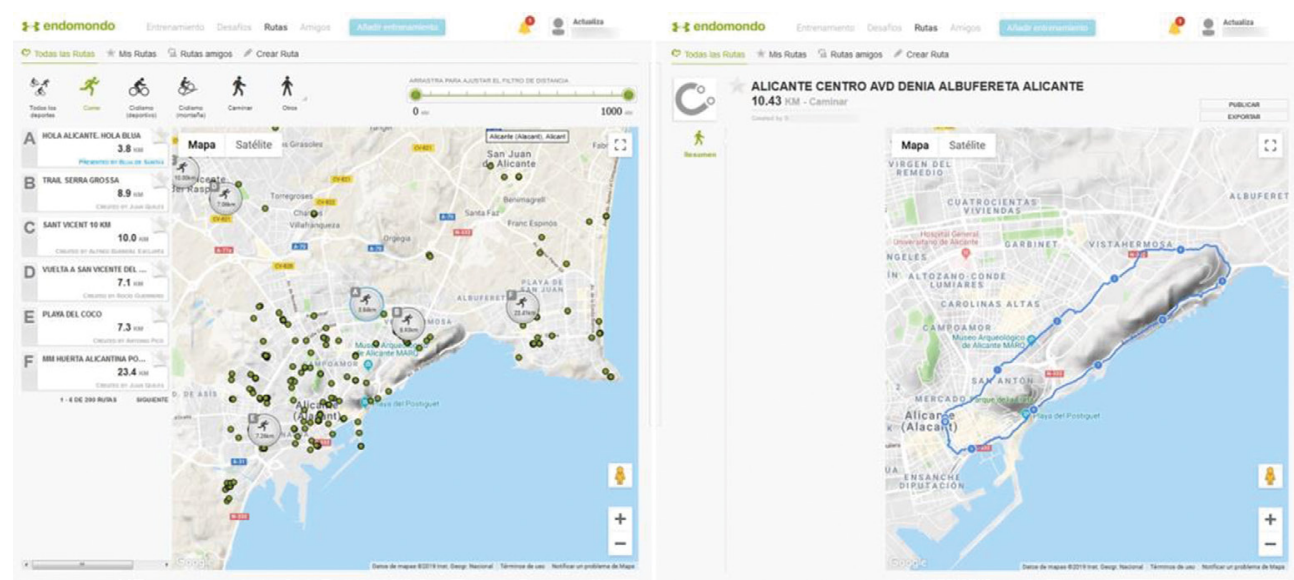

Figure 6: Social network Endomondo. View of different screens of the website: interface for searching and downloading routes (Source: Endomondo).

MapMyRun belongs to the same company as Endomondo, it has common characteristics with Endomondo, especially in the location aspects. It has a website and applications for the main mobile platforms.

The basic functions that it has are as follows:

- Training control: Through the use of Google Maps tools and the location system present in mobile devices, the application is able to obtain some data of exercise sessions that the user can use in order to analyse their performance.

- Recording and sharing of routes obtained with the application by GPS tracking: The creation of routes can be done in the application itself or on the website of the social network. Routes can be saved autonomously and shared immediately.

- Analysis of routes made by other users: The knowledge of these routes offers users the possibility of improving their own sports performance. Fig. 7 shows the information obtained when viewing a route made by another user.

- Creation of activity plans on the different sports disciplines: These plans can be complemented with other functionalities such as live training by a personalized trainer. However, these improvements are not always obtained free of charge, offering only limited functionality.

- This social network also has functions such as events and challenges, many of which are proposed by external service organizers. These functions also make it possible to compare sports activities with other users and share content.

Actually, there is little scientific literature on the social network MapMyRun. More research has been conducted on its sister social network Endomondo. However, an important finding is that, in most cases, the results obtained through the social network MapMyRun have been comparable to those obtained through the social network Strava.

Bearing in mind the similarities with Endomondo (both at company and operational level), it should be noted that MapMyRun focuses more on the person's own training than on the 

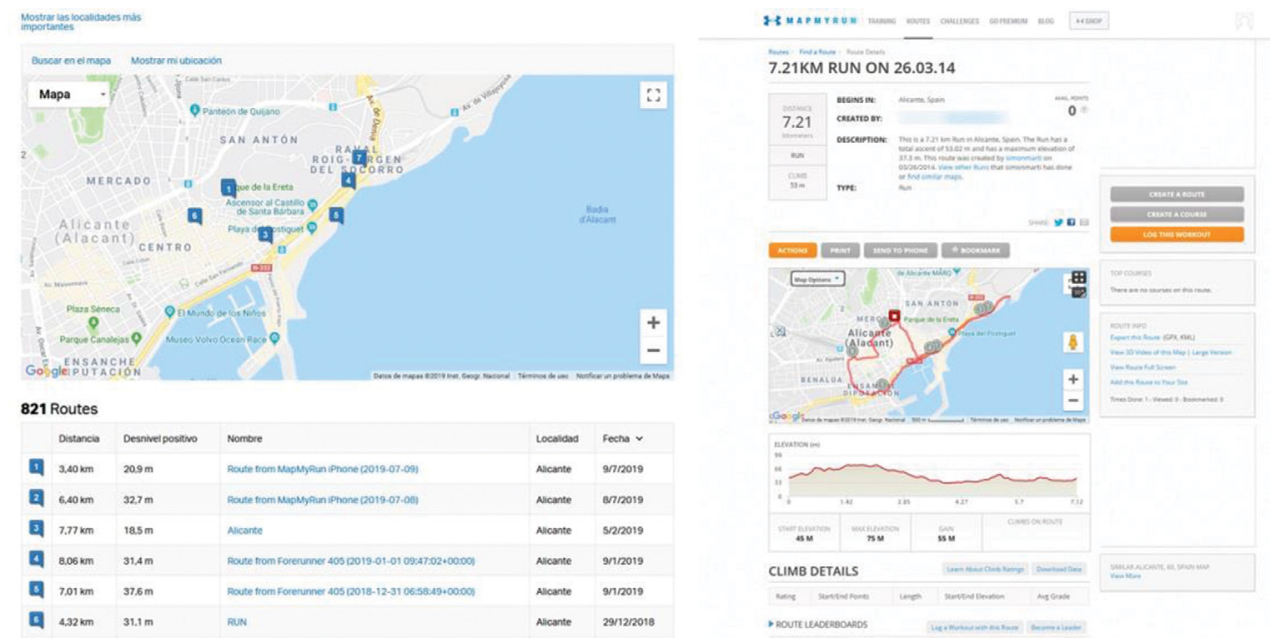

Figure 7: Social network MyMapRun. View of different screens of the website: interface for searching and downloading routes (Source: MyMapRun).

social side of the tool. With this it is possible to achieve better results in the trainings thanks to the existing functions.

In addition, it should be noted that the community of MapMyRun consists of a greater number of users who generate, therefore, a larger set of geolocated data downloadable for free.

\subsection{Social network "Strava"}

The social network Strava is currently one of the most popular and used in the field of sport [18]. In addition, there is an extensive literature related to this social network that consists of a website and its associated application [19,20].

The social network allows users, after selecting the sporting activity they are going to perform, to record the geographic data of the places they pass through while performing the activity and share them within the Strava community. In addition, it is possible to add multimedia components such as photographs related to the activities and it is also possible to share the activities through other social networks such as Facebook or Twitter. Another highlight is the possibility of creating a network of user friends for the purpose of comparison and monitoring of the activity performed.

From the website, it is possible to download and export the activities carried out by other users, regardless of whether or not they belong to the networks of friends. However, not all of these downloads can be made free of charge. So-called "segments" are available. Strava performs a series of calculations to identify the most popular "segments" where athletes practice their activities in the city. Also the "segments" can be created by the users themselves to motivate the competition, although with more exhaustive controls than in other social networks. These "segments" can be obtained from the existing ones in a previously selected geographical environment. In this way, they can be downloaded to the local computer, and analysed with geographic information systems (Fig. 8). In this respect, the variety of formats 

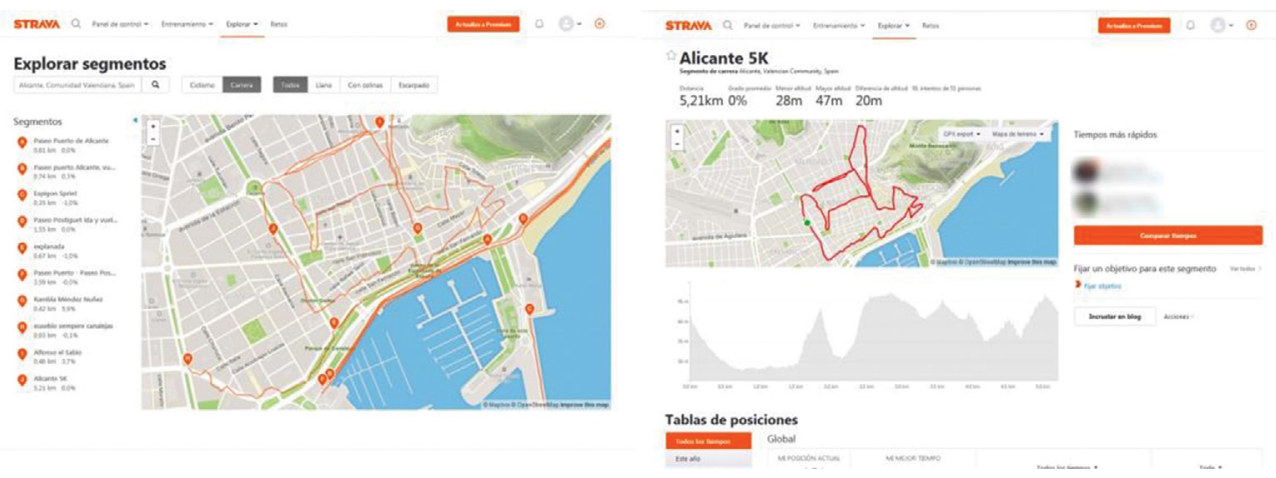

Figure 8: Social network Strava. View of different screens of the website: interface for searching and downloading routes (Source: Strava).

in which information can be downloaded is highlighted, providing flexibility for analysis. It should be noted that Strava offers a very complete documentation of its API.

Also, the existence of the Strava Metro tool [21] should be noted, which analyses the data that Strava obtains through its application in order to provide information such as time spent on streets, and popular or avoidable routes. Obviously, with this tool at the disposal of professionals, it is possible to improve certain aspects of some disciplines, such as urban planning or that of transport engineering [22]. However, Strava Metro is not a free service.

On the other hand, Strava's social approach is clearly perceived. In fact, the interface of its application is divided into two different parts: the one corresponding to exercise and activity, in which it is possible to register all the sports activities carried out; and the one that offers the means to contact other users who carry out the same activities in nearby environments, with which it is possible to share different information about the activity being practised. In this way, Strava presents different characteristics with respect to other social networks studied. Not only does it allow the creation of routes that are registered on the Internet, but it also promotes community awareness with a clear desire for social interaction.

In the field of urban planning, quantitative and qualitative studies are as important, so taking into account only isolated data is not an option. This qualitative information makes it possible to gain a better understanding of citizens' needs, preferences and behaviour patterns.

\section{COMPARATIVE ANALYSIS AND DISCUSSION OF THE ADVANTAGES AND DISADVANTAGES OF SPORT SOCIAL NETWORKS FOR URBAN PLANNING}

Once these eight reference social networks have been described, this section discusses the advantages and disadvantages that make these sports networks an opportunity to move towards smarter, more participatory and inclusive urban planning.

With respect to the social network Atleto, in comparison with the rest of the networks studied, this network does not present a priori advantages to advance towards a smarter urban planning. The main reason is due to the lack of geo-location capacity of the sports activities that it includes, such as running, cycling and walking, among other activities. With the same criteria, the social network Gotzam does not offer users the possibility of registering routes through their own mobile application, nor tools to edit them. Gotzam only offers them the 
possibility of downloading other geolocated routes related to events. However, these geolocated routes do not contain user information, so it is not possible to know the actual routes that users take. Knowing the places visited by users of this type of social networks, through websites and/or their associated applications, is essential to improve the planning and design of cities. That is why it is determined that this social network does not present a priori advantages to move towards a smarter urban planning.

For the rest of the social networks of sport studied, and depending on the characteristics of geographical treatment of the data offered by them, the following considerations are highlighted.

It is evident that the support that each developer provides to social networks in the form of valid information that can be downloaded and analysed has a decisive influence. That is why certain social networks, such as RunKeeper, do not have advantages when it comes to studying the most popular places and routes chosen by citizens. The social network RunKeeper, both within the application and on the website itself, operates with a route system that is more adapted to the person doing the training than to the comparison between different trainings carried out by the users themselves. The routes obtained are isolated within the same training and it is not possible to obtain multiple routes from different users for analysis. This means that only all the routes of the user who performs them can be visualized. In addition, these routes cannot be downloaded, so their study is considerably difficult. This is the main reason why it is determined that the RunKeeper social network does not present any a priori advantages for urban planning either.

The social network Sports Tracker can be considered as a case similar to the social network Runkeeper, because it has historically been specialized in collecting data from users in order for them to check their performance. In addition, Sports Tracker has a major disadvantage, the rigidity when processing geolocated data. It is only possible to download data from complete routes and not from places where several routes can pass at the same time, so it is impossible to get a comprehensive view of the activity that occurs in a particular area of study.

With regard to the social network Runtastic and in relation to obtaining geolocated routes for analysis, it should be noted that, despite what has been said in previous sections, there is no API for downloading data. In this way, the Runtastic social network does not offer the user the possibility of downloading the data separately. It is obligatory to download the complete routes, without the possibility of classification of any kind.

The Endomondo social network allows users to download routes in GPX format by selecting each one of these routes from among those existing in a previously determined graphic window (specific geographical environment), choosing between the different sports activities such as running, cycling, walking, skating, etc. In addition to the geolocated routes that can be downloaded for free, this social network informs of the length of each route, as well as the number of times this route is followed. The routes are created by private users and they can have a greater or lesser number of followers. However, a large number of followers does not imply that the route has been covered by them. This data therefore represents a measure of the activity of users on the social network (on the Internet) but does not reflect the sporting activities that actually take place in the city.

Likewise, the social network MyMapRun allows users to locate existing routes in the environment of a given location. In addition to geolocated routes that can be downloaded in GPX format for free, this social network informs of the length of each route and the date it was created, allowing chronological studies. In addition, you can download a CSV file with information on the total ascent distance of the route and the elevation of each point of the route. 
As in the social network Endomondo, the routes are created by private users and they can have a greater or lesser number of visits on the Internet. However, this data does not reflect the greater or lesser sporting activity that actually takes place in that specific environment of the city. This data only represents the activity of Internet users.

Both social networks, Endomondo and MapMyRun, since they allow users to download geolocated routes, are valid data sources to identify and analyse the routes and places preferred by citizens to practice their daily sports in the city. Therefore, both social networks have their interest in the field of urban planning, especially in decision-making processes, allowing the agents responsible for intervening in the city to take into account, as a priority, the needs and preferences of citizens. However, an important issue is the amount of data that these social networks offer free of charge. As an example, for the geographical area of the city of Valencia in Spain (longitude: -0.3773900; latitude: 39.4697500), MapmyRun offers more than 6,500 routes while Endomondo offers less than 800 routes. Consequently, the social network MyMapRun offers an important advantage in this respect.

In any case, both networks allow the analysis of particular routes created by users, although these are individualized systems that do not provide classifications, rankings or comparisons when the same routes are also travelled by other users. However, the social network Strava does establish these relationships. Once users finish their sporting activity, it is compared and classified with the rest of similar activities carried out by other users. Strava's large community, its classification tables and comparison "segments" give the social network a very advanced potential.

In the field of urban planning, it is very important to have data that reflect the greater or lesser sporting activity that takes place in a specific environment of the city. This knowledge of citizens' preferences facilitates the agents responsible for intervening in the city's decisionmaking processes. In this sense, Strava is a valuable source of data by allowing the downloading of "segments" in which a more or less elevated activity has been previously identified by passing several routes through that particular "segment". Thus, Strava offers free downloads of geolocated "segments" in GPX format and information on both the number of users who travel (although each user makes a different route) and the number of times that the "segment" is covered by the set of users. In addition, Strava offers other data on the difficulty of the "segment", its length, etc. The download of "segments", although not of complete routes, is permitted free of charge by selecting each of these "segments" from among those existing in a graphic window (specific geographical environment) determined previously.

\section{CONCLUSIONS}

In this paper, a series of eight relevant sport social networks in this specific field have been analysed. The study concludes that three of these social networks offer great advantages to move towards smarter, more participatory and inclusive urban planning. Of all the opportunities offered by these sport social networks, this paper has focused on those that offer services free of charge. The social network Endomondo and, more importantly, the social network MyMapRun, allow urban planners to have knowledge of the places and routes that urban athletes choose and prefer for their daily sporting activity. Based on this information, it is possible to characterize these places and establish criteria and priorities for intervention in the city, based directly on the citizens' own experience, i.e. their needs and preferences. On the other hand, the social network Strava provides additional information related to the intensity of the use of urban public space. With this data, urban planners can identify which of all the places preferred by citizens to practice sport in the city are really the most popular. 
As a future work of this research group, it is proposed the application of geolocated data generated by citizens and retrieved from these social networks of sport for free, through the development of a series of case studies in real cities.

\section{ACKNOWLEDGEMENTS}

This work has been funded by the Conselleria de Educación, Investigación, Cultura y Deporte, of the Community of Valencia, Spain, within the program of support for research under project $\mathrm{AICO} / 2017 / 134$.

\section{REFERENCES}

[1] Landry, C., The changing face of urban planning: towards collaborative and creative cities. Human Smart Cities: Rethinking the Interplay Between Design and Planning, Springer, pp. 239-250, 2016.

[2] Hanzl, M., Potential of the Information Technology for the Public Participation in the Urban Planning. Geoinformatics for the Natural Resources Management, Nova Science Publishers: New York, pp. 475-498, 2009.

[3] Mueller, J., Lu, H., Chirkin, A., Klein, B. \& Schmitt, G., Citizen Design Science: A strategy for crowd-creative urban design. Cities, 72, pp. 181-188, 2018.

[4] Social network, "Atleto" website, www.atletosports.com/.

[5] Social network, "Gotzam" website, www.gotzam.com/.

[6] Social network, "Runkeeper" website, www.runkeeper.com/.

[7] Szark-Eckardt, M., Mobile applications as a tool conditioning health of young generation. AIP Conference Proceedings, 2040(1), pp. 070004, 2018.

[8] Martinez-Nicolas, A., Muntaner-Mas, A. \& Ortega, F.B., Runkeeper: a complete app for monitoring outdoor sports. British Journal of Sports Medicine, 51(21), 1560-1561, 2017.

[9] Stragier, J., Vanden Abeele, M. \& De Marez, L. Recreational athletes' running motivations as predictors of their use of online fitness community features. Behaviour \& Information Technology, 37(8), 815-827, 2018.

[10] Social network, "Sports Tracker" website, www.sports-tracker.com/.

[11] Ferrari, L. \& Mamei, M. Identifying and understanding urban sport areas using Nokia Sports Tracker. Pervasive and Mobile Computing, 9(5), pp. 616-628, 2013.

[12] Social network, "Runtastic" website, www.runtastic.com/.

[13] Antón, A.M. \& Rodríguez, B.R., Runtastic PRO app: an excellent all-rounder for logging fitness. British Journal of Sports Medicine, 50(11), 705-706, 2016.

[14] Social network, "Endomondo" website, www.endomondo.com/.

[15] Vickey, T.A., Ginis, K.M., Dabrowski, M. \& Breslin, J.G., Twitter classification model: The ABC of two million fitness tweets. Translational Behavioral Medicine, 3(3), 304311, 2013.

[16] Fioravanti, A., Cursi, S., Elahmar, S., Gargaro, S., Loffreda, G., Novembri, G. \& Trento, A. Visualizing and Analising Urban Leisure Runs by Using Sports Tracking Data. Proceedings of the 35th International Conference on Education and Research in Computer Aided Architectural Design in Europe (eCAADe), vol. I, pp. 533-540, 2017.

[17] Social network, "MyMapRun" website, www.mymaprun.com/.

[18] Social network, "Strava" website, www.strava.com/. 
[19] Hochmair, H.H., Bardin, E. \& Ahmouda, A., Estimating bicycle trip volume for MiamiDade county from Strava tracking data. Journal of Transport Geography, 75, 58-69, 2019.

[20] Sun, Y. \& Mobasheri, A., Utilizing Crowdsourced data for studies of cycling and air pollution exposure: a case study using Strava Data. International Journal of Environmental Research and Public Health, 14(3), 274, 2017.

[21] "Strava Metro" website, https://metro.strava.com/.

[22] Lee, K. \& Sener, I.N., Understanding potential exposure of bicyclists on roadways to traffic-related air pollution: findings from El Paso, Texas, Using Strava Metro Data. International Journal of Environmental Research and Public Health, 16(3), 371, 2019. 